\title{
Mixed input and output orientations of Data Envelopment Analysis with Linear Fractional Programming and Least Distance Measures
}

\author{
Qaiser Farooq Dar ${ }^{1, *}$, Tirupathi Rao Padi ${ }^{1}$ and Arif Muhammad Tali ${ }^{1}$ \\ ${ }^{1}$ Department of Statistics, Pondicherry University, India.
}

(Received: 17 April 2016; Accepted: 24 October 2016)

\begin{abstract}
Data Envelopment Analysis (DEA) is an optimization technique to evaluate the efficiency of Decision-Making Units (DMU's) together with multiple inputs and multiple outputs on the strength of weighted input and output ratios, where as Linear fractional programming is used to obtain DEA frontier. The efficiency scores of DMU obtained through either input orientation or output orientation DEA model will provide only local optimum solution. However, the mixed orientation of input and output variables will provide the global optimal solution for getting the efficient DMUs in DEA. This study has proposed the relationships of a mixed orientation of input and output variables using fractional linear programming along with Least-Distance Measure (LDM). Both constant returns to scale (CRS) and variable returns to scale (VRS) are considered for the comparative study. .
\end{abstract}

Keywords Linear Fractional Programming, Data Envelopment Analysis, Decision Making Units, Mixed-orientation, Least-Distance Measure.

AMS 2010 subject classifications 90C15, 68C05

DOI: $10.19139 /$ soic.v4i4.225

\section{Introduction}

The objective function involving the ratio of two programming problems is the fractional programming. If both numerator and denominator have linear functions of decision variables then such programming is called linear fractional programming, otherwise it is nonlinear fractional programming. The generalization of linear fractional programming is also referred as fractional programming problems (FPP). We need FPP for the situations like (i) maximizing efficiency of the production processes such as maximizing the ratio of virtual output (a linear combination of outputs) to virtual input (a linear combination of inputs) (ii) maximizing the rate of returns to scale on investment (profit to capital) or (iii) maximization of return on risk, etc. DEA is a most powerful optimization technique to take the challenges of efficiencies like Technical efficiency, scale efficiency, allocate efficiency, economic efficiency as well as scope and super efficiency. Many profit and non-profit organizations make use of DEA optimization technique for evaluating and benchmarking the relative efficiencies of different DMUs in the organization.

There is evidence that the FPP is assuming either the ratio of objective functions or the ratio of constraints until 1989 [4]. There are applications where a single ratio is to be maximized or minimized while in other problems the objective function consists of a sum of fractions. Some kind of minimization of input to output was proposed [17], the systematic approaches to this problem was published by Chanes and Cooper [7]. Some

*Correspondence to: Qaiser Farooq Dar (Email: qaiserdea@gmail.com). Department of Statistics, Pondicherry University. Kalapet pondicherry, India (605014).

ISSN 2310-5070 (online) ISSN 2311-004X (print)

Copyright (C) 2016 International Academic Press 
more publications were due to $[22,24,23,27,14]$. The transformation through modification of the feasible set in order to convert the fractional program into a linear program was mentioned by Chanes and Cooper [7]. A large number of problems in the management science directly or indirectly depend on the fractional programs. A more recent comprehensive survey on DEA studies are found in [22]. Charnes, Cooper, and Rhodes [8] introduced a non-parametric optimization technique for evaluates the efficiency of DMU known as DEA which is a special case of FPP. DEA is a very flexible method of comparing the efficiency performance of various decision-making units utilizing the multiple inputs to produce multiple outputs [16] Initially, DEA maximizing the ratio of virtual output (a linear combination of outputs) by virtual input (a linear combination of inputs)[8]. The original CCR model was applicable only to technologies characterized by constant returns to scale. The transformations of linear fractional DEA into LPP model was proposed by [18].The conventional DEA modeling where estimating efficiency values, only for the specific DMU. However an optimal decision is possible only when there is the full information about slacks variables also. The weakness of conventional DEA-model was improved by Joe Zhu see [15]. The maximum efficient score is unity in standard DEA-model. If efficient score is unity, then we can conclude that the specific DMU is full efficient. But in case of small number of DMUs, the efficient set can contain almost all DMUs. In such cases, for the further classification of efficient DMUs the super efficiency model is very useful see [21]. The super efficiency measure is important for ranking of efficient DMU's see [26]. Then slack based measure is applied in case of super efficiency see [11]. More recently, DMUs are assumed as the black box process, where the inefficiency of inefficient DMUs can be identified by dividing into stages, for measuring the efficiency as whole, as well as for each stage independently by using conventional DEA methodology. For two stages see [12], for three stages see[2] and for decomposition of efficiency into networking DEA-model see [13].

DEA has become very popular with more than 40,000 publications in last four decades by over 2,500 authors [10].Empirical analysis of the performance of universities typically takes the form of estimating cost functions with the focus on economies of size and scope or an analysis of efficiency using data envelopment analysis [3].Duality has only established a link between multiplier and envelopment DEA- models [19].Efficiency analysis is performed not only to estimate the current level of efficiency but also provided information about how to remove inefficiencies [9]. Least-Distance Measure (LDM) is a technique which provides the efficiency measure as well as relevant benchmarking information. The LDM define the strongly efficient set first and then calculate the least distance benchmark from the evaluated DMU [5].

In the aforesaid beck drop, this paper is an attempt to utilize the duality concept in FPP for solving the DEAmodels, and explore the duality in DEA for evaluation of productive efficiencies of an organization is characterized by CRS and VRS. In addition, the attempt has been made to explore the concept of input-oriented and outputoriented models for assessing the productive efficiency by mixed-orientation of inputs and outputs in DEA and find the global optimal solution by using LDM. The DEA-model with mixed-orientation has important practical implications which are discussed in this paper which is structured as follows. Section first introduces linear fractional programming (LFP), transformation of LFP into LPP by exploring Chanes, Cooper-transformation, affine transformations and duality in LFP. Section second explains the development of DEA for evaluation of technical efficiencies of organization which is characterized by constant and variable returns to scales with different orientations. Section third includes BCC DEA model with mixed-orientation. Section four deals with the global optimal solution of DEA-model by using LDM approach. The final section discusses data, results and conclusion.

\subsection{Linear Fractional Programming}

The linear fractional programming is the optimization technique dealing with the ratio of two linear functions (or a ratio of two linear programming problems) subject to a set of linear inequalities and non-negativity constraints on the variables. In 1956 linear fractional programming was developed by J.R. Isbell and W.H. Marlow, the problem is solved directly beginning with a basic feasible solution and showing the conditions under which the solution can be improved. The technique followed is similar to the simplex method of linear programming problem (LLP). The general Mathematical form of the linear fractional Programming given by J.R. Isbell and W.H. Marlow is as 
follows:

$$
\begin{aligned}
& \operatorname{Max} z=\frac{c^{\prime} x+\alpha}{d^{\prime} x+\beta} \\
& \text { sub to } \\
& \qquad x \leq b ; x \geq 0
\end{aligned}
$$

where $\mathrm{x}, \mathrm{c}, \mathrm{d}$ are $n \times 1$ vectors, A is a $m \times n$ matrix, $\mathrm{b}$ is a $m \times 1$ vector, c' and d' are the transpose of vector $\mathrm{c}$ and $\mathrm{d}$ and $\alpha, \beta$ are scalar quantities.

When $\left(d^{\prime} x+\beta\right) \neq 0$, the linear fractional programming problem can be expressed as an equivalent linear problem with an additional constraint and an additional variable. The usual simplex method may be then applied to find the optimal solution. There is another approach for solving the linear fractional programming problem (LFPP) is to covert into LPP by using transformation given by Charnes Cooper and States under the assumption that the Feasible Region is non-empty and bounded then the CC transformation is given below:

$$
y=\frac{x}{d^{\prime} x+\beta} \text { and } t=\frac{1}{d^{\prime} x+\beta} \Rightarrow y=x t
$$

The modified problem is in linear from which is feasible for the Simplex Algorithm for solve linear programming problems developed by an American mathematician G.B.Dantziga in 1946. The standard form of the converted problem is as given below.

$$
\begin{aligned}
& \text { Maxz=c } c^{\prime} y+\alpha t \\
& \text { subto } \\
& \qquad \begin{array}{l}
A y-b t \leq 0 \\
\\
d^{\prime} y+\beta t=1 \\
\\
t \geq 0, y \geq 0
\end{array}
\end{aligned}
$$

The model (1.2) is of linear form obtained by Charnes Cooper transformation through denominator normalized linear programming problem. Similarly we can get the numerator normalized linear programming problem for $\left(c^{\prime} x+\alpha\right) \neq 0$ by using the following transformation:

$$
y=\frac{x}{c^{\prime} x+\alpha} \text { and } t=\frac{1}{c^{\prime} x+\alpha} \Rightarrow y=x t
$$

The numerator normalized linear model is given as:

$$
\begin{aligned}
& \text { Max } z=d^{\prime} y+\beta t \\
& \text { subto } \\
& -A y-b t \geq 0 \\
& c^{\prime} y+\alpha t=1 \\
& t \geq 0, y \geq 0
\end{aligned}
$$

The models in (1.2) and (1.3) are LPP's but not in standard from. These LPPs are not feasible for simplex algorithm. In such cases the alternative technique called Duality in linear programming is very useful. The two different dual problems of models are given as. 
Dual Programming Program of Model (1.2)

$\operatorname{Max} \theta$

sub to

$z^{\prime} A+d \theta \geq c$

$-z d+\beta \theta \geq b$

Dual Programming Program of Model (1.3)

$\operatorname{Max} \phi$

sub to

$-z A+c \phi \leq d$

$z^{\prime} b+\alpha \phi \leq b$

$z \geq 0, z \in \Re$ and $\theta \in \Re$

$z \geq 0, z \in \Re$ and $\phi \in \Re$

To preserve co- linearity of linear fractional programming in order to convert into linear programming problem is called Affine Transformation gave by Euler in 1748.

$$
y=\frac{x}{\phi\left(d^{\prime} x+\beta\right)} \text { and } t=\frac{1}{\phi\left(d^{\prime} x+\beta\right)} \Rightarrow y=x t
$$

By using the affine transformation in the linear fractional programming given in the model (1.1) become:

$$
\begin{array}{r}
\text { Max } z=\phi\left(c^{\prime} x+\alpha t\right) \\
\text { subto } \\
A y-b t \leq 0 \\
d^{\prime} y+\beta t=\frac{1}{\beta} \\
t \geq 0, y \geq 0
\end{array}
$$

Alternative setting in the affine transformation for numerator normalized is shown below:

$$
y=\frac{x}{\theta\left(c^{\prime} x+\alpha\right)} \text { and } t=\frac{1}{\theta\left(c^{\prime} x+\alpha\right)} \Rightarrow y=x t
$$

Substitute affine transformation with by above settings in the model (1.1) is called denominator normalized affine transformation and equivalent LPP which given below:

$$
\begin{aligned}
& \text { Min } z=\theta\left(d^{\prime} y+\beta t\right) \\
& \text { sub to } \\
& \text { - Ay+bt } \geq 0 \\
& c^{\prime} y+\alpha t=\frac{1}{\theta} \\
& t \geq 0, y \geq 0
\end{aligned}
$$

The two different Dual problems of models (1.6) and (1.7) are shown in (1.8) and (1.9).

$$
\begin{gathered}
\operatorname{Min} \frac{\theta}{\phi} \\
\text { subto } \\
z^{\prime} A+d \theta \geq c \phi \\
-z^{\prime} b+\beta \theta=\alpha \phi \\
z \geq 0
\end{gathered}
$$


Similarly, the following programming problem is Dual problem of model(1.7).

$$
\begin{array}{cc}
\operatorname{Max} \frac{\phi}{\theta} & \\
\text { subto } & \\
- & z^{\prime} A+c \phi \leq d \theta \\
& z^{\prime} A+\alpha \phi=\beta \theta \\
& z \geq 0
\end{array}
$$

Since models (1.8) and (1.9) are identical and thus can be written as in the following programming problem.

$$
\begin{array}{ll}
\operatorname{Min} \frac{\theta}{\phi} & \\
\text { subto } & \\
& z^{\prime} A-c \phi+d \theta \geq 0 \\
& z^{\prime} b+\alpha \phi-\beta \theta \leq 0 \\
& \theta \geq 0, \phi \neq 0 \text { and } z \geq 0
\end{array}
$$

Since again model (1.8) is the dual of the model (1.6). The weak duality theorem states that if $\mathrm{x}$ is a feasible solution for the primal minimization problem and $\mathrm{y}$ is a feasible solution for the dual maximization problem, then duality implies $g(x) \leq f(y)$; where $f$ and $g$ are the objective functions for primal-dual problems respectively This yield as:

$$
\phi\left(c^{\prime} y+\alpha t\right) \leq \frac{\theta}{\phi} \Rightarrow \frac{c^{\prime} x+\alpha}{d^{\prime} x+\beta} \leq \frac{\theta}{\phi}
$$

Similarly, because (1.9) is the dual of (1.7) then the below result is true:

$$
\theta\left(d^{\prime} y+\beta t\right) \geq \frac{\phi}{\theta} \Rightarrow \frac{c^{\prime} x+\alpha}{d^{\prime} x+\beta} \leq \frac{\theta}{\phi}
$$

Therefore, equation (1.10) is a dual fractional programming of model (1.1) and the relation from (1.1) to (1.10) in shown in the following table;

\begin{tabular}{|l|l|l|l|l|l|}
\hline \multicolumn{5}{|c|}{ Relationship from model (1.1) to model (1.10) } \\
\hline Problem & $\begin{array}{l}\text { Numerator } \\
\text { Normalization }\end{array}$ & $\begin{array}{l}\text { Denominator } \\
\text { Normalization }\end{array}$ & $\theta=1$ & $\phi=1$ & linear fractional form \\
& $(1.7)$ & $(1.6)$ & $(1.3)$ & $(1.2)$ & $(1.1)$ \\
Primal & $(1.9)$ & $(1.8)$ & $(1.5)$ & $(1.4)$ & $(1.10)$ \\
Dual &
\end{tabular}

\section{Data Envelopment Analysis (DEA)}

DEA can be formulated from the LFPP. Thus DEA is particular form of FPP and hence is a LPP based technique for evaluating the efficiency of DMU. The two type of models used in DEA on the basis of the production processes is CCR-model for CRS production processes and BCC-model for VRS production processes with two different types of orientations namely input orientation and output orientation. These sections discussed the mixed-orientation in the situation of CRS and VRS and compare the results with LDM. 


\subsection{Constant Returns to Scale Model (CRS)}

Let $x_{i j}$ and $y_{r j}$ denotes $i^{\text {th }}$ input; $i=1,2,3, \ldots m$ and $r^{\text {th }}$ output; $r=1,2,3, \ldots s$ respectively of the $j^{t h} D M U ; j=1,2,3, \ldots n$. The CCRmodel for calculating the efficiency of $D M U_{k}$ under the assumption of CRS. The Mathematical formulation of CCR, in general, is given as:

$$
\begin{aligned}
E_{k}^{c c r}= & \operatorname{Max} \frac{\sum_{r=1}^{s} u_{r} y_{r k}}{\sum_{i=1}^{m} v_{i} x_{i k}} \\
\text { sub to } & \\
& \frac{\sum_{r=1}^{s} u_{r} y_{r j}}{\sum_{i=1}^{m} v_{i} x_{i j}} \leq 1 ; j=1,2,3, \ldots n . \\
& u_{r} \geq 0 ; r=1,2,3, \ldots \text { s and } v_{i} \geq 0 ; i=1,2,3, \ldots m .
\end{aligned}
$$

Where $u_{r}$ and $v_{i}$ are the unknown weights given to the inputs and outputs respectively.

The mathematical model (2.1.1) is in the fractional form has an infinite number of solutions. In order to avoid fractional form, we are using transformation given by Charnes and Cooper. There is two type of setting based on the denominator normalization and numerator normalization. The denominator normalization is known as inputoriented of CCR model and involves setting:

$$
\begin{aligned}
& t=\frac{1}{\sum_{i=1}^{m} v_{i} x_{i k}}, \mu_{r}=t u_{r} \text { and } \nu_{i}=t v_{i} \Rightarrow \\
& \quad \sum_{r=1}^{s} \mu_{r} y_{r j}=\frac{\sum_{r=1}^{s} u_{r} y_{r j}}{\sum_{i=1}^{m} v_{i} x_{i k}} \text { and } \sum_{i=1}^{m} \nu_{i} x_{i j}=\frac{\sum_{i=1}^{m} v_{i} x_{i j}}{\sum_{i=1}^{m} v_{i} x_{i k}}
\end{aligned}
$$

By using the transformation $(\mathrm{Ta})$ in the mathematical model (2.1.1), the modified model is input-oriented CCRDEA model given by Charnes, Cooper, and Rhodes[8].

$$
\begin{aligned}
E_{k}^{c c r}= & \operatorname{Max} \sum_{r=1}^{s} \mu_{r} y_{r k} \\
\text { subto } & \\
& \sum_{i=1}^{m} \nu_{i} x_{i k}=1 \\
& \sum_{r=1}^{s} \mu_{r} y_{r j}-\sum_{i=1}^{m} \nu_{i} x_{i j} \leq 0, j=1, \ldots n \\
& u_{r}, v_{i} \geq \epsilon \quad r=1, \ldots, s . \text { and } i=1, \ldots, m .
\end{aligned}
$$

Where $\mu_{r}$ and $\nu_{i}$ are the unknown weights given to the inputs and outputs respectively.

Similarly, numerator normalization involves the setting:

$$
\begin{aligned}
& t=\frac{1}{\sum_{r=1}^{s} u_{r} y_{r k}}, \mu_{r}=t u_{r} \text { and } \nu_{i}=t v_{i} \Rightarrow \\
& \quad \sum_{r=1}^{s} \mu_{r} y_{r j}=\frac{\sum_{r=1}^{s} u_{r} y_{r j}}{\sum_{r=1}^{s} u_{r} y_{r k}} \text { and } \sum_{i=1}^{m} \nu_{i} x_{i j}=\frac{\sum_{i=1}^{m} v_{i} x_{i j}}{\sum_{r=1}^{s} u_{r} y_{r k}}
\end{aligned}
$$


Output-oriented CCR model is given as:

$$
\begin{aligned}
E_{k}^{c c r}= & \operatorname{Min} \sum_{i=1}^{m} \nu_{i} x_{i k} \\
\text { sub to } & \\
& \sum_{r=1}^{s} \mu_{r} y_{r k}=1 \\
& \sum_{r=1}^{s} \mu_{r} y_{r j}-\sum_{i=1}^{m} \nu_{i} x_{i j} \leq 0, j=1, \ldots n \\
& u_{r}, v_{i} \geq \epsilon, r=1, \ldots, \text { s. and } i=1, \ldots, m .
\end{aligned}
$$

The models (2.1.2) and (2.1.3) are in multiplier form of CCR model. By using the concept of Duality in the linear programming, we will get envelopment from of (2.1.2) and (2.1.3). Thus the Envelopment from input-oriented of CCR model showing in equation (2.1.2) is given below:

$$
\begin{aligned}
& E_{k}^{c c r}=\operatorname{Min} \theta_{k} \\
& \text { subto } \\
& \qquad \sum_{j=1}^{n} y_{r j} \lambda_{j}-s_{r}^{+}=y_{r k} ; r=1, \ldots s \\
& \\
& \quad \sum_{j=1}^{n} x_{i j} \lambda_{j}+s_{i}^{-}=\theta_{k} x_{i k} ; i=1, \ldots m \\
& s_{r}^{+} \geq 0, s_{i}^{-} \geq 0 \text { and } \lambda_{j} \geq 0 ; j=1, \ldots n .
\end{aligned}
$$

Similarly, envelopment from output-oriented of CCR model showing in equation (2.1.3) is given below:

$$
\begin{aligned}
& E_{k}^{c c r}=\operatorname{Max} \phi_{k} \\
& \text { subto } \\
& \qquad \sum_{j=1}^{n} y_{r j} \lambda_{j}-s_{r}^{+}=\phi_{k} y_{r k} ; r=1, \ldots s \\
& \\
& \quad \sum_{j=1}^{n} x_{i j} \lambda_{j}+s_{i}^{-}=x_{i k} ; i=1, \ldots m \\
& s_{r}^{+} \geq 0, s_{i}^{-} \geq 0 \text { and } \lambda_{j} \geq 0 ; j=1, \ldots n .
\end{aligned}
$$

Another, transformation to convert the fractional form into linear form by applying using affine transformation with setting:

$$
\begin{aligned}
t= & \frac{1}{\phi_{k} \sum_{i=1}^{m} v_{i} y_{i k}}, \mu_{r}=t u_{r} \text { and } \nu_{i}=t v_{i} \Rightarrow \\
& \sum_{r=1}^{s} \mu_{r} y_{r j}=\frac{\sum_{r=1}^{s} u_{r} y_{r j}}{\phi_{k} \sum_{i=1}^{m} v_{i} x_{i k}} \text { and } \sum_{i=1}^{m} \nu_{i} x_{i j}=\frac{\sum_{i=1}^{m} v_{i} x_{i j}}{\phi_{k} \sum_{i=1}^{m} v_{i} x_{i k}}
\end{aligned}
$$


The envelopment from of Input-oriented CCR model is given below:

$$
\begin{aligned}
E_{k}^{c c r}= & \text { Max } \phi_{k} \sum_{r=1}^{s} \mu_{r} y_{r k} \\
\text { sub to } & \\
& \sum_{i=1}^{m} \nu_{i} x_{i k}=\frac{1}{\phi_{k}} \\
& \sum_{r=1}^{s} \mu_{r} y_{r j}-\sum_{i=1}^{m} \nu_{i} x_{i j} \leq 0, j=1, \ldots n . \\
& \mu_{r} \geq 0 ; r=1, \ldots, s . \\
& \nu_{i} \geq 0 ; i=1, \ldots, m .
\end{aligned}
$$

Similarly, affine transformation for output-orientation CCR envelopment model:

$$
\begin{aligned}
& t=\frac{1}{\theta_{k} \sum_{r=1}^{s} u_{r} y_{r k}}, \mu_{r}=t u_{r} \text { and } \nu_{i}=t v_{i} \Rightarrow \\
& \quad \sum_{r=1}^{s} \mu_{r} y_{r j}=\frac{\sum_{r=1}^{s} u_{r} y_{r j}}{\theta_{k} \sum_{r=1}^{s} u_{r} y_{r k}} \text { and } \sum_{i=1}^{m} \nu_{i} x_{i j}=\frac{\sum_{i=1}^{m} v_{i} x_{i j}}{\theta_{k} \sum_{r=1}^{s} u_{r} y_{r k}}
\end{aligned}
$$

Output-oriented CCR envelopment model given as:

$$
\begin{aligned}
E_{k}^{c c r}= & \text { Min } \theta_{k} \sum_{i=1}^{m} \nu_{i} x_{i k} \\
\text { sub to } & \\
& \sum_{r=1}^{s} \mu_{r} y_{r k}=\frac{1}{\theta_{k}} \\
& \sum_{r=1}^{s} \mu_{r} y_{r j}-\sum_{i=1}^{m} \nu_{i} x_{i j} \leq 0, j=1, \ldots n . \\
& \mu_{r} \geq 0 ; r=1, \ldots, s . \\
& \nu_{i} \geq 0 ; i=1, \ldots, m .
\end{aligned}
$$

Dual of modal (2.1.6) and (2.1.7) can be written as:

$$
\begin{aligned}
E_{k}^{c c r}= & \operatorname{Min} \frac{\theta_{k}}{\phi_{k}} \\
\text { subto } & \\
& \sum_{j=1}^{n} y_{r j} \lambda_{j}-s_{r}^{+}=\phi_{k} y_{r k} ; r=1, \ldots s \\
& \sum_{j=1}^{n} x_{i j} \lambda_{j}+s_{i}^{-}=\theta_{k} x_{i k} ; i=1, \ldots m \\
& s_{r}^{+} \geq 0, s_{i}^{-} \geq 0, \phi_{k} \neq 0 \text { and } \lambda_{j} \geq 0 ; j=1, \ldots n .
\end{aligned}
$$


Where $s_{r}^{+}$and $s_{i}^{-}$are input and output slacks.

If $\phi_{k}=1$ then the model (2.1.8) is input- oriented CCR envelopment model, and if $\theta_{k}=1$ then, the model (2.1.8) is output-oriented CCR envelopment model. Since, we know that $\operatorname{Min}\left(\frac{1}{\phi_{k}}\right)$ is same as $\operatorname{Max}\left(\phi_{k}\right)$. By the definition of CCR-efficiency for input-oriented CCR envelopment model. if $\theta_{k}=1$. Then the DMU under evaluation is CCR-efficient, otherwise DMU is inefficient. In the same manner, if $\left(\frac{\theta_{k}}{\phi_{k}}=1\right)$ then the envelopment model (2.1.8) envelopment model with mixed-orientation is efficient. Thus if $\left(\frac{\theta_{k}^{*}}{\phi_{k}^{*}}=1\right)$ then neither current input level can be reduced nor current output level can be expended. This indicates that $D M U_{k}$ is on the frontier. Otherwise, $D M U_{k}$ is dominated by the frontier.

The concept of efficiency given Pareto-Koopmans states that a $D M U_{k}$ under evaluation is efficient if,

1. $\left(\theta_{k}^{*} / \phi_{k}^{*}\right)=1$.

2. All slacks are zero. i.e., $s_{r}^{+}=0, s_{i}^{-}=0 \forall i, j \in \mathbb{Z}^{+}$

If one of the slack is non-zero, then $D M U_{k}$ under evaluation is weakly efficient. This means $D M U_{k}$ can improve by reducing the current level of input and expend the outputs. The identification of possible input excesses and output shortfalls can be improved by solving the mixed-oriented CCR envelopment model (2.1.8) for two phases. In the first phase, we are getting the optimal feasible solution of mixed-oriented CCR envelopment mode. In the second phase, we are using the knowledge of optimal solution and solve for all slacks. Phase Second of CCR envelopment model is given below;

$$
\begin{aligned}
& \operatorname{Max}\left[\sum_{i=1}^{m} s_{i}^{-}+\sum_{j=1}^{s} s_{r}^{+}\right] \\
& \text {subto } \\
& \qquad \sum_{j=1}^{n} y_{r j} \lambda_{j}-s_{r}^{+}=\phi_{k} y_{r k} ; r=1, \ldots s \\
& \qquad \sum_{j=1}^{n} x_{i j} \lambda_{j}+s_{i}^{-}=\theta_{k} x_{i k} ; i=1, \ldots m \\
& s_{r}^{+} \geq 0, s_{i}^{-} \geq 0 \text { and } \lambda_{j} \geq 0 ; j=1, \ldots n .
\end{aligned}
$$

Thus, the envelopment model (2.1.8) and (2.1.9) represent a two-phase of DEA process with mixed-orientation. From equation (2.1.8) and (2.1.9) we will get Two phase of CCR envelopment model as shown below equation $(2.1 .10)$.

$$
\begin{aligned}
E_{k}^{c c r}= & \operatorname{Min} \frac{\theta_{k}}{\phi_{k}}-\epsilon\left[\sum_{i=1}^{m} s_{i}^{-}+\sum_{j=1}^{s} s_{r}^{+}\right] \\
\text {subto } & \\
& \sum_{j=1}^{n} y_{r j} \lambda_{j}-s_{r}^{+}=\phi_{k} y_{r k} ; r=1, \ldots s \\
& \sum_{j=1}^{n} x_{i j} \lambda_{j}+s_{i}^{-}=\theta_{k} x_{i k} ; i=1, \ldots m \\
& s_{r}^{+} \geq 0, s_{i}^{-} \geq 0, \phi_{k} \neq 0 \text { and } \lambda_{j} \geq 0 ; j=1, \ldots n .
\end{aligned}
$$

If $\phi_{k}$ is taking as a constant then model (2.1.10) is input-oriented CCR envelopment model. If $\theta_{k}$ is taking as a 
constant then model (2.1.10) is output-oriented CCR envelopment model. In the phase $2^{\text {nd }}$, we are subtracting $\epsilon\left[\sum_{i=1}^{m} s_{i}^{-}+\sum_{j=1}^{s} s_{r}^{+}\right]$for input-oriented and adding the same factor for output-oriented in the objective function. Where the $\epsilon$ is non-Archimedean constant Charnes [8]. In phase first we solve envelopment model (2.1.10) for $\theta_{k}$ or $\phi_{k}$ by putting one of them as fixed to one. In phase $2^{\text {nd }}$, we are using the estimated value of both $\theta_{k}^{*}$ and $\phi_{k}^{*}$ to solve the model (2.1.10) for $\left(\lambda_{j}, s_{r}^{+}\right.$and $s_{i}^{-} \forall i, j$ and $\left.r\right)$. The $D M U_{k}$ is efficient if $\theta_{k}^{*}=1$ and $\phi_{k}^{*}=1$ and all slacks are zero. Otherwise, $D M U_{k}$ is considered inefficient.

\subsection{Variable Returns to Scale Model (VRS)}

As previously stated, the CRS data envelopment model assumes that the DMU's are operating at an optimal scale. This model permits a measure of global technical efficiency to be obtained without variations in returns to scale. In the real world however, this optimal behavior is often precluded by some factors such as in the imperfect competition and constraints in finance etc. To take this circumstance into account Banker Charnes and Cooper [6] have extended DEA to the case of VRS. This model distinguishes between pure technical efficiency (TE) and scale efficiency (SE), and identifies if increasing, decreasing or constant returns to scale are present. As a consequence, the assumptions of CRS envelopment model has changed by adding one more constraint know as convexity constraint.

$$
\begin{aligned}
& E_{k}^{b c c}=\operatorname{Min} \theta_{k} \\
& \text { sub to } \\
& \qquad \sum_{j=1}^{n} y_{r j} \lambda_{j}-s_{r}^{+}=y_{r k} ; r=1, \ldots s \\
& \quad \sum_{j=1}^{n} x_{i j} \lambda_{j}+s_{i}^{-}=\theta_{k} x_{i k} ; i=1, \ldots m \\
& \quad \sum_{j=1}^{n} \lambda_{j}=1 ; j=j=1, \ldots n . \\
& \quad \lambda_{j} \geq 0, s_{r}^{+} \geq 0 \text { and } s_{i}^{-} \geq 0 .
\end{aligned}
$$

The multiplier form of BCC-model is obtained by using the CC-transformation as shown in the previous section in equation $(T a)$ and $(T b)$ for input-oriented and output -oriented BCC-models respectively. By exploring the concept of duality in Data Envelopment Analysis given by Charnes and Cooper [6], for converting the multiplier form into the envelopment form. The efficiency criterion is given by Pareto-Koopmans [1] states that a $D M U_{k}$ under evaluation is efficient if,

\section{BCC model with orientations.}

- $E_{k}^{b c c}=1 \Rightarrow\left(\theta_{k}=1\right) \rightarrow$ input - oriented BCC-model.

- $E_{k}^{b c c}=1 \Rightarrow\left(\phi_{k}=1\right) \rightarrow$ output-oriented BCC-model.

2. All slacks are zero. i.e., $s_{r}^{+}=0, s_{i}^{-}=0 \forall i, j \in \mathbb{Z}^{+}$.

Both the conditions (1) and (2) must be satisfied, then only $D M U_{k}$ is fully technical efficient and if, condition (1) only is satisfied then the $D M U_{k}$ is said weakly efficient. Otherwise, if condition (1) also not satisfies the $D M U_{k}$ is said to be inefficient. 


\section{BCC Data Envelopment Analysis Model with mixed-orientation}

The BCC variable returns to scale DEA model given by Banker,Charnes and Cooper [6] is applicable for both input-oriented and output-oriented respectively. In the input-oriented BCC-model, where we tries to minimizing the input level (resources) in order to produce the same level of output (production). Similarly, in the output-oriented BCC-model we are maximize output level (production) by utilizing fixed amount of inputs (resources). But there are many combinations of inputs and outputs lying between input and output orientations coming under mixedoriented which are more satiable for the optimal efficiency. In the mixed-orientation we are trying to improve the efficiency by changing both input and outputs of $D M U_{k}$ under evaluation. For the graphical representation of mixed-orientation see figure 1 .

The $D M U A, B, C, D, E$ and $F$ are efficient in the constant returns to scale DEA-model, while

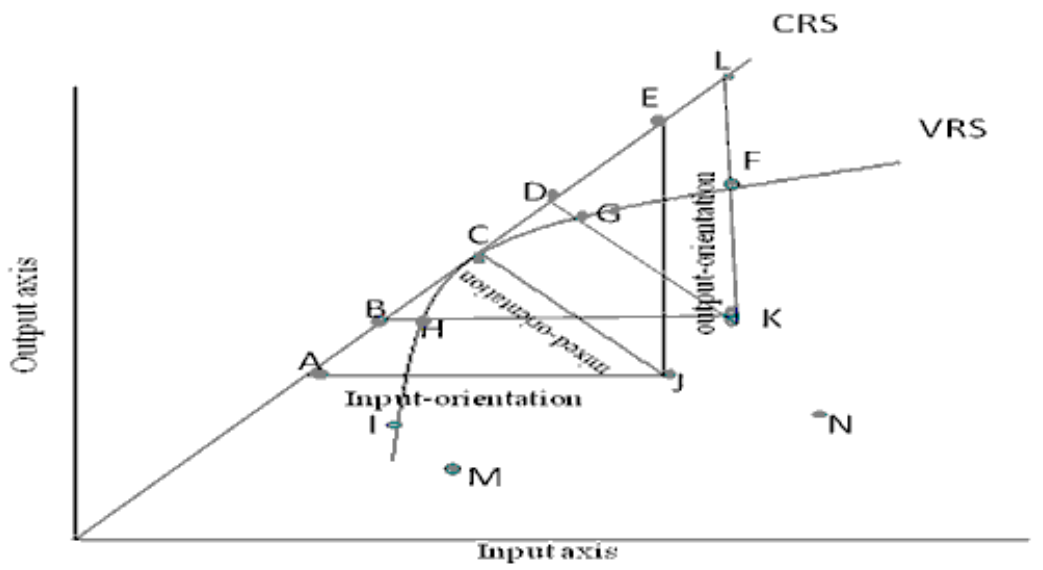

Figure 1. Input, Output and Mixed orientations in Constant and variable Return scale production.

$D M U H, C, G$ and $F$ are efficient in the variable returns to scale DEA-model and other DMUs are inefficient. $D M U C$ is efficient in both the situations. In CRS, the value input orientation is same as the value of output orientation. But it is not true VRS as shown $D M U_{j}$ and $D M U_{K}$ in Fig.1. The inefficient $D M U s$ can achieve their targets (efficiency) by using any of the orientation, but mixed-orientation is only orientation through which the inefficient $D M U s$ gets the global optimization of value efficiency.

\subsection{Mathematical formulation of BCC-model with mixed-orientation}

In both the orientations. viz, input-oriented and output-oriented give only the local optimization. But global optimization has to be achieved in a mixed orientation. The convexity constraint $\sum_{j=1}^{n} \lambda_{j}=1 ; j=1, \ldots n$. imposed in the envelopment form of BCC (variable returns to scale) DEA model [6], can also be imposed to obtain the envelopment form of a mixed-oriented variable returns to scale DEA model (3.1) from the mixed-oriented constant returns to scale DEA model (2.8). The envelopment form of BCC-model with mixed-orientation is as follows:

$$
E_{k}^{b c c}=\text { Minimise } \frac{\theta_{k}}{\phi_{k}}
$$


sub to

$$
\begin{aligned}
& \sum_{j=1}^{n} y_{r j} \lambda_{j}-s_{r}^{+}=\phi_{k} y_{r k} ; r=1, \ldots s \\
& \sum_{j=1}^{n} x_{i j} \lambda_{j}+s_{i}^{-}=\theta_{k} x_{i k} ; i=1, \ldots m \\
& \sum_{j=1}^{n} \lambda_{j}=1 ; j=j=1, \ldots n . \\
& s_{r}^{+} \geq 0, s_{i}^{-} \geq 0, \phi_{k} \neq 0 \text { and } \lambda_{j} \geq 0
\end{aligned}
$$

where $E_{k}^{b c c}$ is the efficiency of $D M U_{k}$.

The efficiency score of inefficient DMUs in CCR-model with mixed-orientation lying between the input and output orientations efficiency scores. The constancy of the value of $\theta_{k} / \phi_{k}$ does not apply for variable returns to scale DEA-model because $\theta_{k}$ (value of input-orientation) in BCC-model does not equal to $1 / \phi_{k} \quad\left(\phi_{k}\right.$ value of output-orientation). Since the projected value of inefficient $D M U_{k}$ is lying on the line segment joining the values of two orientations. Since the value of $\theta_{k}$ lies between $\left(\theta_{k i} \leq \theta_{k} \leq 1\right)$ where $\theta_{k I}$ is the input oriented value of $K^{t h}-D M U$, and value of $\phi_{k}$ lies between $\left(1 \leq \phi_{k} \leq \phi_{k o}\right)$. Where $\phi_{k o}$ is the output-oriented value of $K^{t h}-D M U$ in the BCC-model. The value of $\left(\theta_{k} / \phi_{k}\right)$ is calculated by using the input-oriented and outputoriented BCC-models or put $\phi_{k}=1$ in (3.2.1). While $\frac{1}{\phi_{k}}$ is the efficiency score of output BCC DEA model.

\section{Least Distance Measure (LDM)}

Both the basic models CCR [8] under the assumption of CRS and BCC [6] under the assumption of VRS are applicable either an input-orientation (i.e. indicate that an inefficient DMU is made efficient through the proportional reduction of its inputs while its outputs proportions are held constant), or output-orientation (i.e. an inefficient unit is made efficient through the proportional increase of its outputs, while the inputs proportions remain unchanged). In CRS situation, the efficiency value of $D M U^{\prime} s$ using input-oriented DEA-model is same as efficiency value of $D M U^{\prime} s$ using output-oriented DEA-model. But it is not true in BCC (VRS) DEA model. Since though input-oriented and output-oriented DEA-models of an inefficient $D M U^{\prime} s$ achieves only local optimization efficiency score, while global optimization efficiency score achieves only by using mixed-oriented (varying both inputs level and outputs level) simultaneously in the DEA-models. In CRS DEA-model, the efficiency value of inefficient $D M U^{\prime} s$ using mixed-orientation is lying between other two orientations. But in VRS, the efficiency score of inefficient $D M U^{\prime} s$ lies between other two orientations graphically, but not numerically. This section addresses Least-Distance measure (LDM) with mixed-orientation of inefficient $D M U s$.

The set of observation satisfying the Pareto-efficiency conditions and their convex combination is defined as strongly efficient set $E_{t}$, such that,

$$
\begin{gathered}
E_{t}=\left[\left(x^{t}, y^{t}\right) / \operatorname{Max}\left[\sum_{r=1}^{s} s_{r}^{+}+\sum_{i=1}^{m} s_{i}^{-}\right]\right]=0 \\
\text { subto } \\
s_{i}^{-}=x_{i t}-\sum_{j=1}^{n} x_{i j} \lambda_{j} ; i=1, \ldots m .
\end{gathered}
$$




$$
\begin{aligned}
& s_{r}^{+}=\sum_{j=1}^{n} y_{r j} \lambda_{j}-y_{r t} ; r=1, \ldots s \\
& \sum_{j=1}^{n} \lambda_{j}=1 ; \lambda_{j} \geq 0 \forall j=1, \ldots n .
\end{aligned}
$$

where $X_{i j}$ is the $i^{t h}$ input, $y_{r j}$ is the $r^{t h}$ output of $j^{t h}$ DMU and $E_{t}$ is a strongly efficient set of $k D M U s$ [9]. The classified DEA-efficient $D M U s$ as strongly (extremely) efficient if and only if, the maximum number of the nonzero optimal virtual multiplier for a $D M U$ in the set is equal to the dimension of performance space. The concept of least-distance is derived from the extremely (strongly) efficient set $E_{t}$, and it is more suitable in a primal space rather than in dual space.

Least-Distance measure is given by Baek, and Lee, [5] the objective function of Least-Distance Measure converts the distance between the evaluated $D M U\left(x_{k}, y_{K}\right)$ and the strongly efficient set $E_{t}$ (targeted set) is described as follows:

$$
\begin{aligned}
& \theta_{k}=\operatorname{Max}\left[1-\frac{1}{m+s}\left\{\sum_{i=1}^{m}\left(\frac{x_{i}^{t}-x_{i}^{k}}{R_{i}^{-}}\right)^{2}+\sum_{r=1}^{s}\left(\frac{y_{r}^{t}-y_{r}^{k}}{R_{r}^{+}}\right)^{2}\right\}^{\frac{1}{2}}\right] \\
& \text { sub to } \\
& \left.\qquad x^{t}, y^{t}\right] \in E_{t} \\
& \text { where } R_{i}^{-}=\max _{j}\left\{x_{i j}\right\}-\min _{j}\left\{x_{i j}\right\} . \\
& R_{r}^{+}=\max _{j}\left\{y_{r j}\right\}-\min _{j}\left\{y_{r j}\right\} . \\
& x_{i j} \text { and } y_{r j} \text { arethe } i^{\text {th }} \text { inputand } r^{\text {th }} \text { outputof } j^{\text {th }} \text { DMUrespectively. }
\end{aligned}
$$

$x^{k}$ and $y^{K}$ are inputs and outputs of the $k^{t h}$ inefficient DMU (DMU under evaluation), $k \in j=1,2, . . n .\left(x^{t}, y^{t}\right)$ are inputs and outputs of $t^{t h}$ strongly efficient DMU (targeted DMU), $t \in[j=1,2, . ., n]$.

The first and foremost activity in LDM is to define the strongly efficient set of $D M U s$ and then evaluate the inefficient $D M U$ by calculating the least- distance benchmark between them. The limitation associated with both orientation in the CCR and BCC envelopment models overcome by using the LDM and it satisfy the conditions of good efficiency measure [20] which are defined as follows;

- (P1). ( $\theta=1)$ if and only if, $D M U_{k}$ is fully efficient. i.e., $\theta$ asymptotically converges to 0 if and only if $D M U_{k}$ is fully inefficient, such that $0 \leq \theta \leq 1$.

- (P2). $\theta$ is strongly monotonic.

- (P3). $\theta$ satisfying the translation invariant property.

- (P4). $\theta$ is unit invariant.

\subsection{Least- Distance measure (LDM) algorithm}

The algorithm of LDM includes four steps.

Step 1:- Solve the additive DEA model for each $D M U$ and categorize each $D M U$ as either Pareto efficient or 
inefficient. The Pareto efficient DMU $\left(X^{t}, y^{t}\right)$ have zero as the optimal value of additive DEA and defined set $E_{t}$, such that $E_{t}$ satisfy model (4.1). For each Pareto inefficient $D M U^{*}$, and go to next step.

Step 2:- Make combination composed of $(m+s)$ components of set $E_{t}$, where $m$ and $s$ are the number of inputs and outputs respectively. If the number of components of set $E_{t}$ equal to $t$, then $\left({ }^{t} C_{m+s}\right)$ combinations are available [20].

Step 3:- For each combination compute the quadratic equation as shown below and obtain optimal solution as $\left(x^{*}, y^{*}\right)[5]$

$$
\operatorname{Min}\left\{\sum_{i=1}^{m}\left(\frac{x_{i}^{t}-x_{i}^{k}}{R_{i}^{-}}\right)^{2}+\sum_{r=1}^{s}\left(\frac{y_{r}^{t}-y_{r}^{k}}{R_{r}^{+}}\right)^{2}\right\}
$$

sub to

$$
\begin{aligned}
& x=X_{t}^{E} \lambda \\
& y=Y_{t}^{E} \lambda \\
& e^{t} \lambda=1 \\
& \lambda \geq 0
\end{aligned}
$$

Where $X_{t}^{E}$ and $Y_{t}^{E}$ is the input and output matrix of $t^{t h}$ combination of set E, $x^{t}=\left(x_{1}, x_{2}, x_{3}, \ldots, x_{m}\right) y^{t}=$ $\left(y_{1}, y_{2}, y_{3}, \ldots, y_{s}\right)$

Step 4:- sort the array of $\left(x^{\prime}, y^{\prime}\right)$ is an increasing order according to the objective of the model (4.1) and solve the additive DEA by adding each $\left(x^{\prime}, y^{\prime}\right)$ one by one. The first $\left(x^{\prime}, y^{\prime}\right)$ evaluated as being additive efficient is defined as $\left(x^{*}, y^{*}\right)$, and then $\left(x^{*}, y^{*}\right)$ is the nearest projection point from $\left(x^{k}, y^{k}\right)$ to the strongly efficient set $E_{t}$. Then,

$$
\theta=1-\frac{1}{m+s}\left\{\sum_{i=1}^{m}\left(\frac{x_{i}^{t}-x_{i}^{k}}{R_{i}^{-}}\right)^{2}+\sum_{r=1}^{s}\left(\frac{y_{r}^{t}-y_{r}^{k}}{R_{r}^{+}}\right)^{2}\right\}^{\frac{1}{2}}
$$

Where $\theta$ becomes the efficiency measure in the Least-Distance Measure [5]. The quadratic model (4.1.1) can be transformed into a linear programming problem.

\section{Empirical illustration}

This section provides an empirical illustration which helps to clarify the difference between CRS and VRS with mixed-orientation of inputs and outputs and comparative advantage of LDM upon the conventional DEA models. The financial data of 24 Islamic Banking institutions [25] is used as an empirical illustration which consists of three inputs and two outputs, out of 24 banks, only 6 banks $(1,4,14,16,18$ and 21) are inefficient in both situations of BCC with CRS and VRS, as shown in (Table 1).

\subsection{Results and Conclusion}

The efficiency value of input and output orientations for inefficient $D M U^{\prime} s$ under the CRS is same. But it not true in general for VRS as shown in the Table 1 under column (4 and 8) for each inefficient $D M U s$. This shows the optimal value of inputs and outputs in the mixed-orientation model is not usually lying between the two orientations. The input and output-oriented DEA models achieved the local optimal solution of efficiency, where as the mixed-orientation DEA model of inefficient DMU's provide the global optimal solution of inputs and outputs 
as shown in (Table 2). The benchmarking information under the input-oriented BCC- Approach is fixed at least one output (either $\triangle y_{1}=0$ or $\triangle y_{2}=0$ or both); where as in mixed-orientation we are able to change both input and output simultaneously as shown in (Table 2) under Least- Distance Measure. The paper focused on least distance between the initial value (given value of inputs and out puts) and benchmarking value (targeted value of inputs and outputs) of inefficient DMUs. The least- distance measure provided the nearest benchmark of inputs and outputs in the inefficient DMUs; as shown in (Table 2) by comparing the $L_{i}$ (input-oriented distance), $L_{o}$ (output-oriented distance) and L (least-distance).

Table I. Results of BCC-Model (CRS and VRS) of inefficient banks using mixed orientation.

\begin{tabular}{||c|c|c|c|c|c|c|c|c||}
\hline \hline \multirow{2}{*}{ DMU's } & \multicolumn{3}{|c|}{ Constant Returns to Scale Results } & \multicolumn{3}{|c||}{ Variable Returns to Scale Results } \\
\cline { 2 - 9 } & $\theta(1)$ & $\phi(2)$ & $\frac{\theta}{\phi}(3)$ & $\frac{\phi}{\theta}(4)$ & $\theta(5)$ & $\phi(6)$ & $\frac{\theta}{\phi}(7)$ & $\frac{\phi}{\theta}(8)$ \\
\hline \multirow{2}{*}{ Bank 1 } & 0.9786 & 1 & 0.9786 & 1.0218 & 0.9407 & 1 & 0.9907 & 1.0094 \\
\cline { 2 - 9 } & 1 & 1.0218 & 0.9786 & 1.0218 & 1 & 1.0888 & 0.9185 & 1.0875 \\
\hline \multirow{2}{*}{ Bank 4 } & 0.5441 & 1 & 0.5441 & 1.8378 & 0.5783 & 1 & 0.5783 & 1.7293 \\
\cline { 2 - 9 } & 1 & 1.8377 & 0.5441 & 1.8378 & 1 & 1.7109 & 0.5845 & 1.7109 \\
\hline \multirow{2}{*}{ Bank 14 } & 0.7621 & 1 & 0.7621 & 1.3122 & 0.7835 & 1 & 0.7835 & 1.2764 \\
\cline { 2 - 9 } & 1 & 1.3121 & 0.7621 & 1.3122 & 1 & 1.2989 & 0.7699 & 1.2989 \\
\hline \multirow{2}{*}{ Bank 16 } & 0.9265 & 1 & 0.9265 & 1.0794 & 0.9269 & 1 & 0.9269 & 1.0789 \\
\cline { 2 - 9 } & 1 & 1.0793 & 0.9265 & 1.0794 & 1 & 1.0792 & 0.9266 & 1.0792 \\
\hline \multirow{2}{*}{ Bank 18 } & 0.9256 & 1 & 0.9259 & 1.0800 & 0.9531 & 1 & 0.9531 & 1.0492 \\
\cline { 2 - 9 } & 1 & 1.0800 & 0.9259 & 1.0800 & 1 & 1.0463 & 0.9557 & 1.0463 \\
\hline \multirow{2}{*}{ Bank 21 } & 0.8866 & 1 & 0.8866 & 1.1279 & 0.9221 & 1 & 0.9221 & 1.0845 \\
\cline { 2 - 9 } & 1 & 1.1279 & 0.8866 & 1.1279 & 1 & 1.0829 & 0.9234 & 1.0829 \\
\hline \hline
\end{tabular}

Note:- $\theta$ is output - oriented value efficiency calculated by putting $\phi=1$ in the model (3.2.1), while $\frac{1}{\phi}$ is input-oriented efficiency value calculated by putting $\theta=1$ in the model (3.2.1). The benchmarking information of inefficient DMUs by using BCC DEA technique and Least-Distance Measure technique, are as shown in (Table 2).

Table II. Comparison of Benchmarking information of DEA -BCC Approach with Least- Distance Measure Approach

\begin{tabular}{|c|c|c|c|c|c|c|c|c|c|c|c|c|c|}
\hline \multirow{2}{*}{ DMU's } & \multicolumn{6}{|c|}{ Input-Oriented BCC- Approach } & \multirow[b]{2}{*}{$L_{o}$} & \multicolumn{6}{|c|}{ Least- Distance Measure Approach } \\
\hline & $\triangle x_{1}$ & $\triangle x_{2}$ & $\triangle x_{3}$ & $\triangle y_{1}$ & $\triangle y_{2}$ & $L_{i}$ & & $\triangle x_{1}$ & $\triangle x_{2}$ & $\triangle x_{3}$ & $\triangle y_{1}$ & $\triangle y_{2}$ & $\bar{L}$ \\
\hline Bank 1 & 76 & 1159 & 464 & 0 & 0 & 1251 & 2474 & 15 & 820 & 2 & 327 & 7 & 883 \\
\hline Bank 4 & 150 & 15675 & 2373 & 4257 & 0 & 16415 & 27066 & 268 & 15355 & 1115 & 4723 & 1031 & 16138 \\
\hline Bank 14 & 705 & 9625 & 1575 & 0 & 0 & 9778 & 19487 & 128 & 8524 & 27 & 3745 & 91 & 9312 \\
\hline Bank 16 & 429 & 7977 & 3656 & 0 & 0 & 8785 & 19132 & 649 & 445 & 184 & 8298 & 731 & 8369 \\
\hline Bank 18 & 969 & 21944 & 1160 & 0 & 0 & 21995 & 36272 & 154 & 20215 & 209 & 5740 & 1050 & 21100 \\
\hline Bank 21 & 490 & 36877 & 2380 & 1380 & 0 & 39463 & 73736 & 906 & 16725 & 1381 & 1069 & 2149 & 33560 \\
\hline
\end{tabular}

Note: $\triangle$ means the change of each variable to remove inefficient, i.e., $\triangle=$ initial -optimal. $\mathrm{L}$ denotes the distance between given value and targeted value; $L_{i}$ and $L_{O}$ are the input and output oriented distances using BCC DEA-model.

\section{Acknowledgement}

Authors are grateful to an anonymous referee of this journal for the most constructive observations, and suggestion, which materially improved this paper. 


\section{REFERENCES}

1. A. Charnes, W.W. Cooper, B. Golany and L. Seiford, Foundations of data envelopment analysis for Pareto-Koopmans efficient empirical production functions, Journal of econometrics, vol. 30, no. 1, pp. 91- 107, 1985.

2. A. Ebrahimnejad, M. Tavana, F.H. lotfi, R. Shahverdi, and M. yousfpour, A three-stage data envelopment analysis model with application to banking industry Measurement, vol. 49, pp. 308- 319, 2014.

3. M. Abbott, and C. Doucouliagos, The efficiency of Australian universities: a data envelopment analysis, Economics of Education review, vol. 22, no. 1, pp. 89-97, 2003.

4. H. Arsham, and A.B. Kahn, A complete algorithm for linear fractional programs, Computers and Mathematics with Applications, vol. 20, no. 7, pp. 11-23, 1990.

5. C. Baek, and J. Lee., The relevance of DEA benchmarking information and the least-distance measure, Mathematical and Computer Modelling, vol. 49, no. 1, pp. 265-275, 2009.

6. R.D. Banker, A. Charnes, and W. W. Cooper, Some models for estimating technical and scale inefficiencies in data envelopment analysis, Management science, vol. 30, no. 9, pp. 1078-11092, 1984.

7. A. Charnes, and W.W. Cooper, Programming with linear fractional functionals, Naval Research logistics quarterly, vol. 9, no. 3-4, pp. 181-186, 1962.

8. A. Charnes, W.W. Cooper, and E. Rhodes, Measuring the efficiency of decision-making units, European journal of operational research, vol. 3, no. 4 pp. 339-351, 1978.

9. A. Charnes, W.W. Cooper, and R.M Thrall, A structure for classifying and characterizing efficiency and inefficiency in data envelopment analysis, Journal of Productivity Analysis, vol. 2, no. 3 pp. 197-237, 1991.

10. A. Emrouznejad, B.R. Parker, and G. Tavares, Evaluation of research in efficiency and productivity: A survey and analysis of the first 30 years of scholarly literature in DEA, Socio-economic planning sciences, vol. 42, no. 3 pp. 151-157, 2008.

11. J. Jablonsky, A slack based model for measuring super-efficiency in data envelopment analysis, Multiple Criteria Decision Making, vol. 5, pp. 101-112, 2006.

12. K. Chiang, and S.N. Hwang, Efficiency decomposition in two-stage data envelopment analysis: An application to non-life insurance companies in Taiwan, European Journal of Operational Research, vol. 185, no. 1 pp. 418-429, 2008.

13. K. Chiang, Efficiency decomposition in network data envelopment analysis with slacks-based measures, Omega, vol. 45, pp. 1-6, 2014.

14. M. Shaban, V. Kavida, and A. Shah, Evaluating Efficiency of Commercial Banks in India: Using DEA Model, Pacific Business Review International, vol. 5, pp. 9-18, 2013.

15. H. Morita, K. Hirokawa, and J. Zhu. A slack-based measure of efficiency in context-dependent data envelopment analysis, Omega, vol. 33 , no. 4 pp. 357-362, 2005.

16. H. Morita, and N.K. Avkiran, Selecting inputs and outputs in data envelopment analysis by designing statistical experiment (; Special Issue; Operations Research for Performance Evaluation), Journal of the Operations Research Society of Japan, vol. 52, no. 2 pp. 163-173, 2009.

17. J.V. Neumann, A model of general economic equilibrium." Readings in the Theory of Growth, Palgrave Macmillan UK, pp. 1-9, 1971.

18. G.C. O'Brien, and L. Wu, Alternative transformations and duality of linear fractional programming, In Operations Research/Management Science at Work (pp. 333-350), Springer US - 2002.

19. K.S. Park, Duality, efficiency computations and interpretations in imprecise DEA, European Journal of Operational Research, vol. 200, no. 1 pp. 289-296, 2010.

20. T. Räty, Efficient facet based efficiency index: A variable returns to scale specification, Journal of Productivity Analysis, vol. 17, no. $1-2$ pp. $62-82,2002$.

21. S.J. Sadjadi, H. Omrani, S. Abdollahzadeh, M.Alinaghian, and M. Hodammadi. A robust super-efficiency data envelopment analysis model for ranking of provincial gas companies in Iran, Expert Systems with Applications, vol. 38, no. 9 pp. 10875-10881, 2011.

22. S. Schaible, Fractional programming, In Handbook of global optimization in Springer US., pp. 495-608, 1995.

23. S. Schaible, and J. Shi, Fractional programming: the sum-of-ratios case, Optimization Methods and Software, vol. 18, no. 2 pp. 219-229, 2003.

24. S. Siegfried, Analyse und Anwendungen von Quotientenprogrammen: e. Beitr. zur Planung mit Hilfe $d$, nichtlinearen Programmierung. Vol. 42, 1978.

25. K. Shahooth, and A.H. Battall, Using data envelopment analysis to measure cost efficiency with an application on Islamic banks, Scientific Journal of Administrative Development, vol. 4, pp. 134-156, 2006.

26. Seiford, M. Lawrence, and J. Zhu., Infeasibility of super-efficiency data envelopment analysis models, INFOR: Information Systems and Operational Research,vol. 37, no. 2 pp. 174-187, 1999.

27. I.M. Stancu-Minasian, Fractional programming: theory, methods, and applications Springer Science and Business Media, vol. 409, 2012. 